\title{
Antibacterial effect of sophoraflavanone G by destroying the cell wall of Enterococcus faecium
}

\author{
Daseul Kim¹, Ki-young Kim ${ }^{1,2, *}$ \\ ${ }^{1}$ Graduate School of Biotechnology, Kyung Hee University, Seocheon, Giheung, Yongin, Gyeonggi-do 446-701, Republic of Korea \\ ${ }^{2}$ College of Life Science, Kyung Hee University, Seocheon, Giheung, Yongin, Gyeonggi-do 446-701, Republic of Korea
}

\begin{tabular}{l}
\hline ARTICLE INFO \\
\hline Received on: $23 / 09 / 2019$ \\
Accepted on: $26 / 06 / 2020$ \\
Available online: $05 / 09 / 2020$
\end{tabular}

\section{Key words:}

E. faecium, sophoraflavanone $\mathrm{G}$, antibacterial activity, natural compound.

\begin{abstract}
Enterococcus faecium has appeared as an important opportunistic pathogen that can cause urinary tract infections, surgical site infections, bacteremia, and endocarditis. Therefore, it is imperative to develop alternative therapeutic methods to treat enterococcal infections. Sophoraflavanone G $\left(5,7,2^{\prime}, 4^{\prime}\right.$-tetrahydroxy-8-lavandulylflavanone, SPF-G) exhibited the strongest antibacterial activity based on the minimum inhibitory concentration values on two E. faecium strains $(6.25$ and $12.5 \mu \mathrm{g} / \mathrm{ml}$ at 24 and 48 hours of treatment, resp.) in the broth microdilution assay among the tested compounds and a remarkable bactericidal effect with a $12.5 \mu \mathrm{g} / \mathrm{ml}$ minimum bactericidal concentration value. Sophoraflavanone G $(12.0 \pm 2.3 \mathrm{~mm}$ inhibition zone for Korean Agricultural Culture Collection, Korea (KACC) 11954 and $11.0 \pm 3.0 \mathrm{~mm}$ for Culture Collection of Antimicrobial Resistant Microbes, Korea (CCARM) 5506) also exhibited the highest susceptibility based on the agar diffusion assay. Membrane-permeabilizing agents with a low dose of sophoraflavanone $G$ synergistically activated anti-E. faecium activity through a $67 \%$ reduction of $E$. faecium growth, and E. faecium-derived peptidoglycans (PGN) blocked the antibacterial activity. These results indicate that sophoraflavanone $\mathrm{G}$ could bind to the bacterial cell wall and induce E. faecium cell wall damage. Transmission electron microscopy (TEM) images of E. faecium treated with sophoraflavanone $\mathrm{G}$ also exhibited cell lysis, followed by leakage of intracellular components, confirming that sophoraflavanone $\mathrm{G}$ has anti-E. faecium activity by binding to the PGN and disrupting the cell wall. This study showed the possible usage of sophoraflavanone $G$ as an effective natural anti-E. faecium compound.
\end{abstract}

\section{INTRODUCTION}

Enterococci commonly reside within the human gastrointestinal and genitourinary tracts and can cause enterococcal bacteremia, infective endocarditis, and urinary tract infection (Graham and Gould, 2002; Morrison et al., 1997; Murdoch et al., 2002; Murray, 1990). Over the last decades, Enterococci isolated by hospital-acquired infections have showed increased intrinsic or acquired antibiotics resistance to the most currently approved agents. Particularly, enterococcal or nosocomial infection caused by resistant strain is life-threatening in humans (Vignaroli et al., 2010).

\section{"Corresponding Author}

Ki-Young Kim, PhD, Department of Genetic Engineering, College of Life Science and Graduate School of Biotechnology, Kyung Hee University, Seocheon, Giheung, Yongin, Gyeonggi-do 446-701, Republic of Korea. E-mail: kiyoung@khu.ac.kr
Among the Enterococci species, Enterococcus faecium has appeared as a serious opportunistic pathogen with major causation of multidrug-resistant enterococcal infection especially in immunocompromised patients. Even though Enterococcus faecalis is isolated more frequently, E. faecium isolates often showed resistance to vancomycin, ampicillin, penicillin, and oxacillin. The drug resistance of E. faecium made that species difficult to be treated, and therefore the incidence of the problem is increased. Approximately $40 \%$ of recent medical deviceassociated infections were due to multidrug-resistant $E$. faecium. Therefore, it is crucial to the development of new antimicrobial agents and alternative therapy to treat multidrug-resistant Enterococci infections (Miller et al., 2015; Vignaroli et al., 2010).

Natural compounds have been referred to as those produced by plants or animals and are investigated because they possess potential bioactive materials that have antimicrobial activity, anticancer activity, and so on (Kim et al., 2004). 
Eleven plant-originated compounds were used to screen the antibacterial activity against E. faecium and found sophoraflavanone $G$ as a compound that has the strongest antibacterial activity among the tested ones.

Sophoraflavanone G, also known as a 5,7,2'4'tetrahydroxy-8-lavabdulyflavanone, is a crenelated flavonoid that can be isolated from the root of Sophora flavescens (Figure 1). Sophoraflavanone $\mathrm{G}$ has a variety of biological activities including antibacterial activity against opportunistic bacteria including Streptococcus sobrinus, Streptococcus mutans, and Staphylococcus aureus (Gerber et al., 2009; Hsiang et al., 2001; Orsi et al., 2011; Tsai et al., 2004).

This study presented the antibacterial mechanisms of sophoraflavanone $\mathrm{G}$ to treat $E$. faecium infections. The antimicrobial activity of sophoraflavanone $G$ was obtained by determining the minimum inhibitory concentration (MIC) using the broth microdilution method, and then the antibacterial mechanism of sophoraflavanone $\mathrm{G}$ by disrupting the E. faecium cell wall was investigated by growing in combination with an $E$. faecium-derived peptidoglycan (PGN), detecting the release of UV-absorbing material, and images captured using transmission electron microscopy.

\section{MATERIALS AND METHODS}

\section{Bacterial strains}

Enterococcus faecium (KACC 11954 and CCARM 5506) were purchased from KACC (Korean Agricultural Culture Collection, Korea) or CCARM (Culture Collection of Antimicrobial Resistant Microbes, Korea). The bacterial strains were maintained on Muller-Hinton (MB cell, USA) agar plates and incubated at $37^{\circ} \mathrm{C}$.

\section{Antibacterial activity of sophoraflavanone $G$ against $E$. faecium}

The broth microdilution method was applied to determine the MICs using microplates (Clinical and Laboratory Standards Institute, 2006; Tsai et al., 2004). Enterococcus faecium strains were cultured in Muller-Hinton broth for 24 hours at $37^{\circ} \mathrm{C}$ and then transferred to a 96-well plate of $0.5 \mathrm{McF}$ arland standard. Serial twofold dilutions of sophoraflavanone G (Sigma, St. Louis, MO) were prepared in Muller-Hinton broth. The MIC value was determined as the minimum compound concentration that inhibited bacterial growth after the indicated time. Ampicillin, vancomycin, and streptomycin were used as positive controls.

The minimum bactericidal concentration (MBC) was obtained using a microdilution assay according to the standard with slight modification (Clinical and Laboratory Standards Institute, 2006). After bacteria were cultured in the same manner as the MIC test, E. faecium strains were inoculated onto the agar plates and incubated for 24 hours at $37^{\circ} \mathrm{C}$. MBCs were determined by the lowest concentration of compound which exhibit no viable bacteria colony. The experimental and control groups then were assigned to the wells in triplicate.

\section{Susceptibility test}

Broth inoculums with 0.5 McFarland standard were spread onto sterile Muller-Hinton broth by cotton swabs. The $8 \mathrm{~nm}$ sterile paper discs were impregnated with $100 \mu \mathrm{g} / \mathrm{ml}$ compounds, and the inhibition zones were measured after 18 hours (Climo et al., 1999; Jorgensen and Ferraro, 2009).

\section{Growth curve assay}

The growth curve was obtained using the previously described 96-well microplate. After this, sophoraflavanone G was added to the cells and incubated at $37^{\circ} \mathrm{C}$, for an indicated time (Lee YS, 2015). To assess the anti-E. faecium synergistic effect of sophoraflavanone $\mathrm{G}$ with the membrane-permeabilizing chemical, $3.125 \mu \mathrm{g} / \mathrm{ml}$ of sophoraflavanone $\mathrm{G}$ was added in combination with $0.01 \%$ Triton X-100. Neither compound inhibited bacterial growth (Lim and Strynadka, 2002; Mun et al., 2014, Sasaki et al., 2012).

To analyze the effect of the PGN on anti-E. faecium activity of sophoraflavanone $\mathrm{G}$, bacterial inoculum $\left(\mathrm{OD}_{600}=\right.$ 0.1) was added to the Mueller-Hinton broth containing different concentrations of sophoraflavanone $\mathrm{G}$ and/or PGN and then incubated for 24 hours. The growth of E. faecium was evaluated by measuring $\mathrm{OD}_{600}$ using microplate reader (Mun et al., 2014; Viktoryia and Vitaly, 2014).

\section{Isolation of PGN from cell walls of gram-positive bacteria}

Overnight bacteria cultures were washed twice with saline, and then prewarmed $20 \mathrm{ml}$ of $30 \%$ aqueous phenol was added to the bacteria for 20 minutes at $65^{\circ} \mathrm{C}-68^{\circ} \mathrm{C}$.

After cooling to $20^{\circ} \mathrm{C}$, the mixture was centrifuged 3 times for 10 minutes at 1700 Revolutions per minute (RPM), with the removal of the supernatant after each cycle. At this stage, the noncovalently associated contaminant was removed. The product obtained in the preceding step was stirred at $100^{\circ} \mathrm{C}$ for 3 hours with $300 \mathrm{ml}$ of distilled water and $3 \mathrm{ml}$ of $100 \%$ acetic acid to eliminate trace amounts of lipopolysaccharide (LPS). After the precipitate was washed 3 times with water, dialysis with $0.05 \mathrm{M}$ sodium acetate solution was carried out at $21^{\circ} \mathrm{C} \pm 2{ }^{\circ} \mathrm{C}$ (Desmarais et al., 2014; Furukawa et al., 1993; Kuhner et al., 2014; Viktoryia and Vitaly, 2014).

\section{Detection of release of $U V$-absorbing materials}

The release of UV-absorbing materials was measured using a UV spectrophotometer with slight modifications. Sophoraflavanone $\mathrm{G}$ was applied to E. faecium strains at a final concentration of $0-50 \mu \mathrm{g} / \mathrm{ml}$. Absorbance measurements of the supernatant at $260 \mathrm{~nm}$ and $280 \mathrm{~nm}\left(\mathrm{~A}_{260}\right.$ and $\left.\mathrm{A}_{280}\right)$ were recorded (Septama et al., 2017).

\section{Transmission electron microscopy analysis}

Enterococcus faecium were treated with $6.25 \mu \mathrm{g} / \mathrm{ml}$ of sophoraflavanone $\mathrm{G}$ for 30 minutes. After removal of supernatant, the pellets were fixed with a microscopy grid (Ted Pella). The specimens were analyzed using a transmission electron microscope (JEM-1010, JEOL, Seoul, Korea, 80 kV) (Mun et al., 2014).

\section{Statistical analysis}

All experiments were performed at least three times, and data were expressed as the median with individual data including standard deviation.

\section{RESULTS}

\section{Sophoraflavanone $G$ possesses anti-E. faecium activity}

Antibacterial susceptibility tests of sophoraflavanone $\mathrm{G}$ against $E$. faecium strains were performed based on the standard 
broth microdilution method. The MIC values of sophoraflavanone G against $E$. faecium were $12.5 \mu \mathrm{g} / \mathrm{ml}$ (KACC 11954 and CCARM 5506), but the MIC values of ampicillin and streptomycin used as positive controls were $6.25 \mu \mathrm{g} / \mathrm{ml}$ (KACC 11954 and CCARM 5506), $>100 \mu \mathrm{g} / \mathrm{ml}$ (KACC 11954), and $0.78 \mu \mathrm{g} / \mathrm{ml}$ (CCARM 5506), respectively. Both strains exhibited resistance against vancomycin (MIC value $>100 \mu \mathrm{g} / \mathrm{ml}$ ) (Table 1 ).

The MBC value was obtained by subculturing the samples from the wells in the MIC assays. MBC values were not altered for E. faecium strain $(12.5 \mu \mathrm{g} / \mathrm{ml})$ after 24 hours at $37^{\circ} \mathrm{C}$ following further incubation for 18-24 hours (Table 2).

A bacterial susceptibility test was also performed to determine the antibacterial effect of sophoraflavanone $G$ on the solid agar plate. Antibacterial activity of sophoraflavanone $\mathrm{G}$ was confirmed by measuring the diameters of the zone of growth inhibition, and the highest susceptibility was shown to E. faecium $(12 \pm 2.3 \mathrm{~mm}$ for CCARM 5506; $11 \pm 3 \mathrm{KACC} 11954$ (Table 3)).

To confirm the antibacterial effect of sophoraflavanone $\mathrm{G}$, the growth curve was used, and sophoraflavanone $\mathrm{G}$ exhibited concentration-dependent inhibitory activity against both $E$. faecium strains. Interestingly, $6.25 \mu \mathrm{g} / \mathrm{ml}$ of sophoraflavanone $\mathrm{G}$ completely blocked bacterial growth (Fig. 2).

\section{Sophoraflavanone $G$ synergistically inhibited $E$. faecium growth with membrane-permeabilizing agents}

Enterococcus faecium strain was treated with a combination of sophoraflavanone $\mathrm{G}$ and the membrane- permeabilizing agent Triton X-100 (0.01\%) to determine whether the sophoraflavanone $G$ altered membrane permeability. The nonionic detergents of Triton X-100 were used to increase the permeability of the outer membrane. At the tested concentration, the treatment of single compound did not significantly affect $E$. faecium growth, but the combination of the two did decrease $E$. faecium growth (Fig. 3).

\section{Sophoraflavanone $G$ increased release of $U$-absorbing materials}

Release of UV-absorbing materials was measured using a UV spectrophotometer to confirm cell wall disturbance by sophoraflavanone $\mathrm{G}$. The absorption change indicated material leakage from the cell including proteins and nucleic acids (Septama et al., 2017). The higher absorption value suggested greater cell disruption. Significant increases in absorption at 260 and $280 \mathrm{~nm}$ after treatment with sophoraflavanone $\mathrm{G}$ suggest that sophoraflavanone $\mathrm{G}$ binds to and disrupts the cell wall (Fig. 4).

\section{Sophoraflavanone $G$ inhibited $E$. faecium growth by binding to PGNS}

To determine whether sophoraflavanone $G$ blocks E. faecium growth by binding to PGNs that induce cell wall perturbation, PGNs were added to the culture with sophoraflavanone G.

Table 1. MIC values against E. faecium.

\begin{tabular}{ccccc}
\hline & \multicolumn{2}{c}{ MIC value $(\boldsymbol{\mu g} / \mathbf{m l})$} & \multicolumn{2}{c}{ CCARM 5506 } \\
Compounds & \multicolumn{2}{c}{ KACC 11954 } & 24 hours & 48 hours \\
\hline Ampicillin & 24 hours & 48 hours & 3.125 & 6.25 \\
Vancomycin & 1.56 & 6.25 & 0.78 & 0.78 \\
Streptomycin & $>100$ & $>100$ & $>100$ & $>100$ \\
Acetylshikonin & $>100$ & $>100$ & 12.5 & 12.5 \\
6.8-Diprenylorobol & 25 & 50 & 25 & 50 \\
Kurarinone & 25 & 50 & 25 & 50 \\
Sophoraflavanone G & 25 & 50 & 6.25 & 12.5 \\
Pimaric acid & 6.25 & 12.5 & 100 & 100 \\
Corosolic acid & 100 & 100 & 12.5 & 25 \\
Oleanolic acid & 12.5 & 25 & $>100$ & $>100$ \\
Ursolic acid & $>100$ & $>100$ & $>100$ & $>100$ \\
\hline
\end{tabular}

Table 2:. MBC values of sophoraflavanone $\mathrm{G}$ and ampicillin against E. faecium CCARM 5506.

\begin{tabular}{cccc}
\hline \multicolumn{2}{c}{ MIC $(\mu \mathrm{g} / \mathrm{ml})$} & \multicolumn{2}{c}{ MBC $(\mu \mathrm{g} / \mathrm{ml})$} \\
Sophoraflavanone G & Ampicillin & Sophoraflavanone G & Ampicillin \\
\hline 12.5 & 6.25 & 25 & 50 \\
\hline
\end{tabular}

Table 3:. Inhibition diameters of sophoraflavanone G against E. faecium.

\begin{tabular}{ccc}
\hline \multirow{2}{*}{ Compounds } & \multicolumn{2}{c}{ Inhibition zone (mm) } \\
\cline { 2 - 3 } & KACC 11954 & CCARM 5506 \\
\hline Ampicillin & $18 \pm 1.5$ & $24 \pm 2$ \\
Sophoraflavanone G & $12 \pm 2.3$ & $11 \pm 3$ \\
\hline
\end{tabular}


PGNs dose-dependently blocked the antibacterial activity of sophoraflavanone $\mathrm{G}$ even at low concentrations of 6.25 and $12.5 \mu \mathrm{g} / \mathrm{ml}$. This result suggests that sophoraflavanone $\mathrm{G}$ binds to bacterial PGNs to block bacteria growth (Fig. 5a and b).

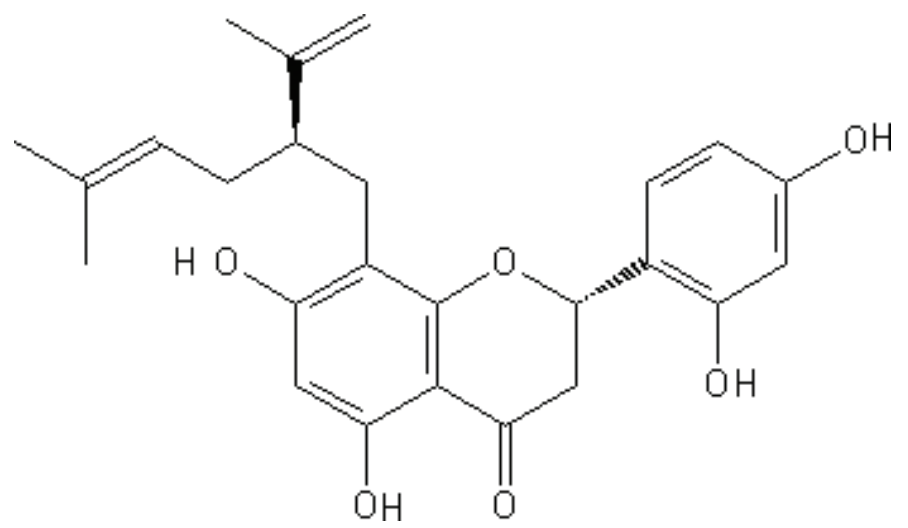

Figure 1. Chemical structure of sophoraflavanone G.

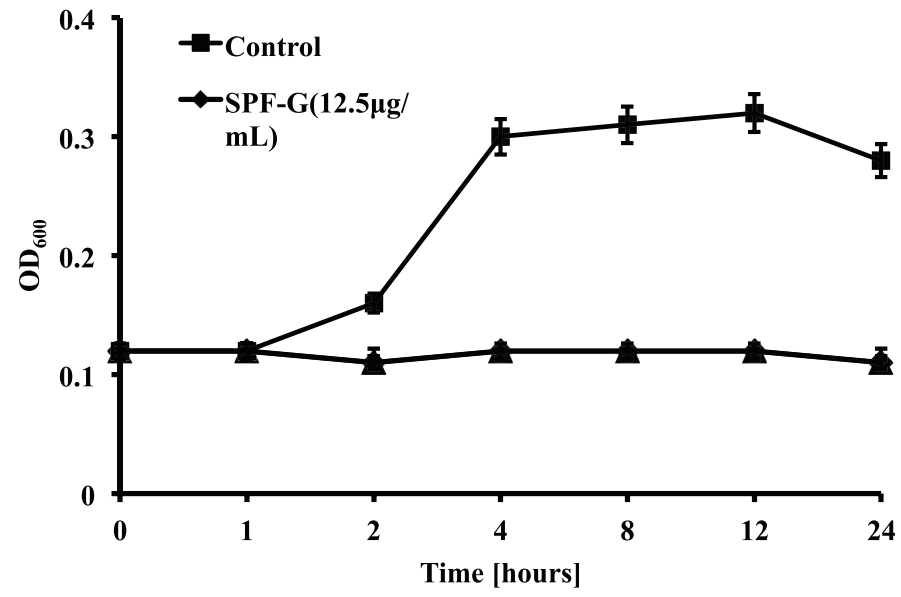

Figure 2. Sophoraflavanone $\mathrm{G}$ inhibited the growth of E. faecium. Control: untreated control E. faecium; SPF-G: sophoraflavanone G $(12.5 \mu \mathrm{g} / \mathrm{ml})$ and sophoraflavanone $\mathrm{G}(6.25 \mu \mathrm{g} / \mathrm{ml})$.

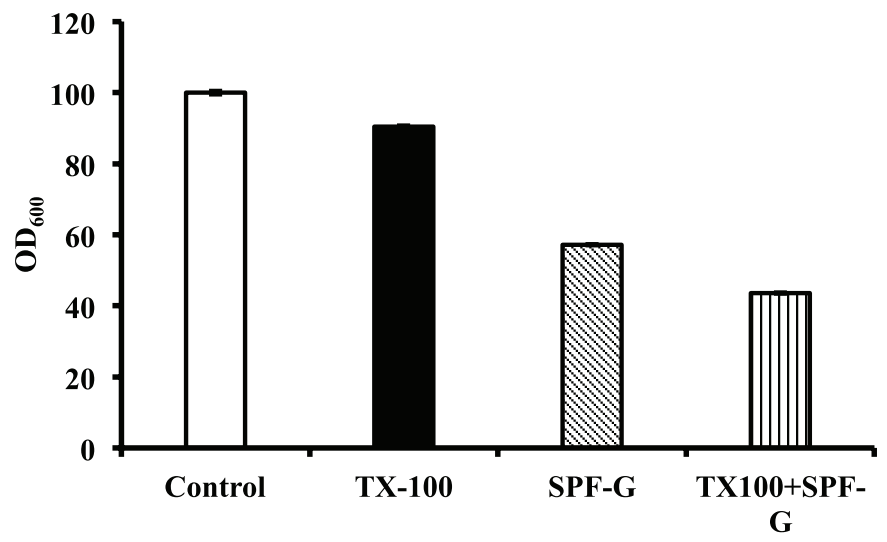

Figure 3. Sophoraflavanone G $(3.125 \mu \mathrm{g} / \mathrm{ml})$ synergistically inhibited growth of E. faecium with membrane-permeabilizing agents. Bacterial viability was determined by absorbance at $600 \mathrm{~nm}$ after incubation for 24 hours with sophoraflavanone G $(3.125 \mu \mathrm{g} / \mathrm{ml})$ and/or $0.01 \%$ Triton X-100. Control: untreated control E. faecium cells, TX-100: $0.01 \%$ Triton X-100, SPF-G: sophoraflavanone G $(3.125 \mu \mathrm{g} / \mathrm{ml})$, and TX-100+SPF-G: $0.01 \%$ Triton X-100 + sophoraflavanone G $(3.125 \mu \mathrm{g} / \mathrm{ml})$.

\section{Transmission electron microscopy}

To confirm whether sophoraflavanone $G$ disrupted E. faecium cells, sophoraflavanone G-treated bacterial cells were examined by TEM. A 24-hours exposure of $6.25 \mu \mathrm{g} / \mathrm{ml}$ of

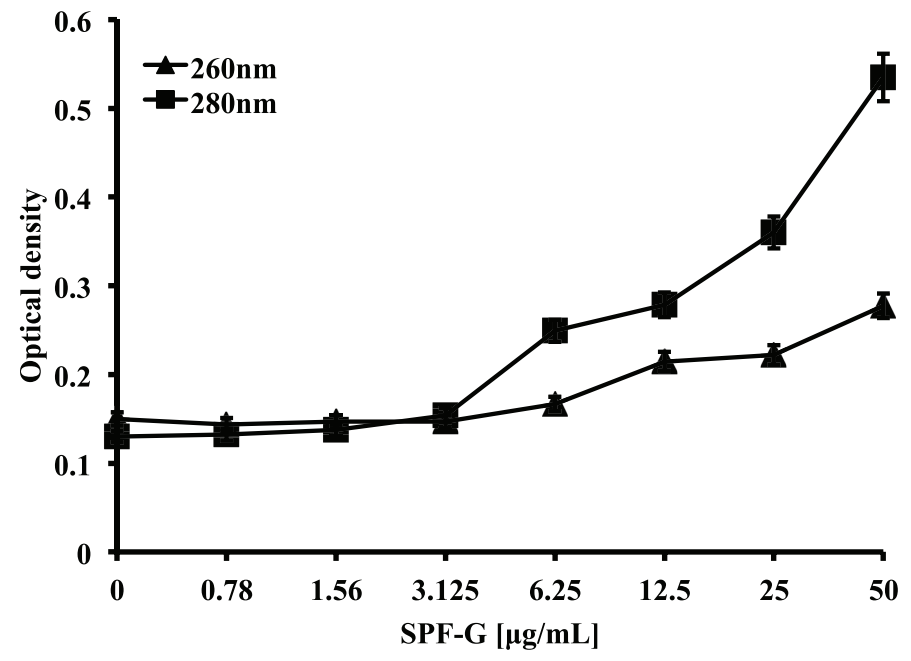

Figure 4. Sophoraflavanone G increased the leakage of cell materials.

(A)

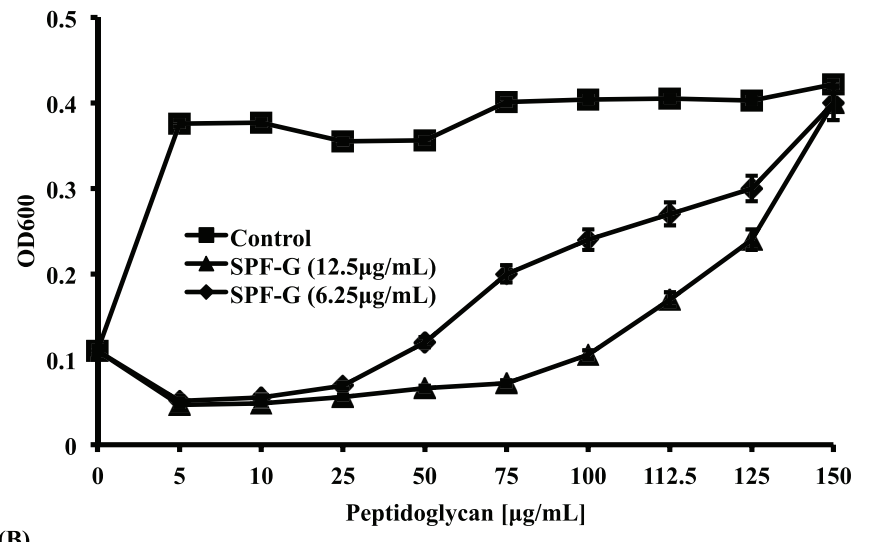

(B)

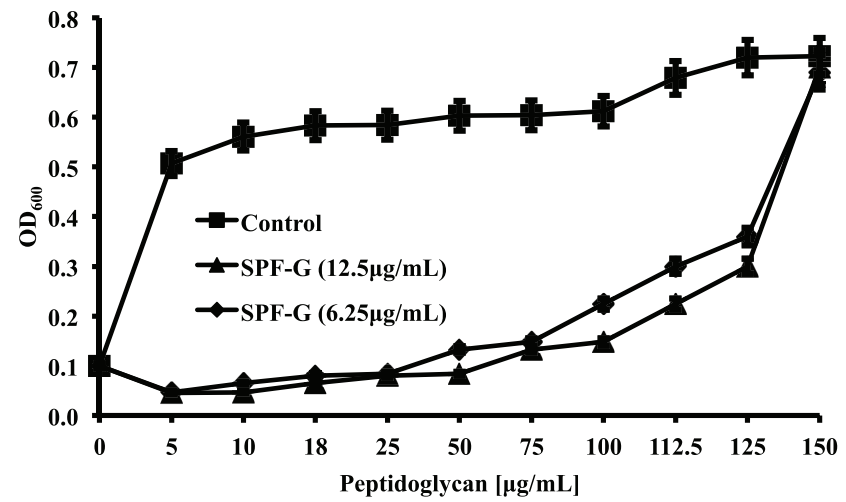

Figure 5. E. faecium cell wall $\mathrm{PGN}$ inhibited the antibacterial activity of sophoraflavanone $\mathrm{G}(6.25 \mu \mathrm{g} / \mathrm{ml})$ against $E$. faecium. (A) Control: E. faecium; SPF-G: sophoraflavanone G $(12.5 \mu \mathrm{g} / \mathrm{ml})$ and sophoraflavanone $\mathrm{G}(6.25 \mu \mathrm{g} / \mathrm{ml})$.(B) Control: S. aureus; SPF-G: sophoraflavanone $\mathrm{G}(12.5 \mu \mathrm{g} / \mathrm{ml})$ and sophoraflavanone $\mathrm{G}(6.25 \mu \mathrm{g} / \mathrm{ml})$. 


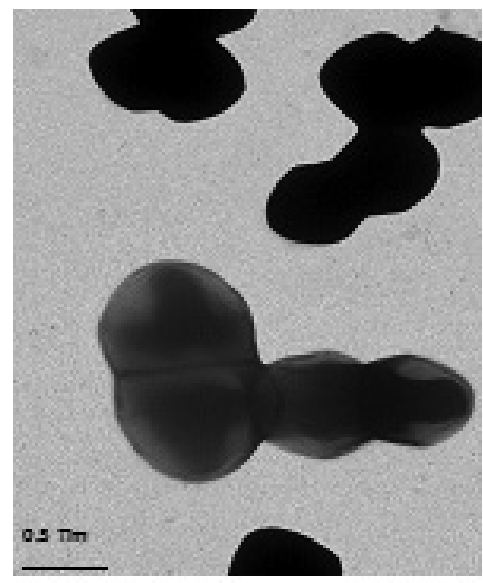

(A)

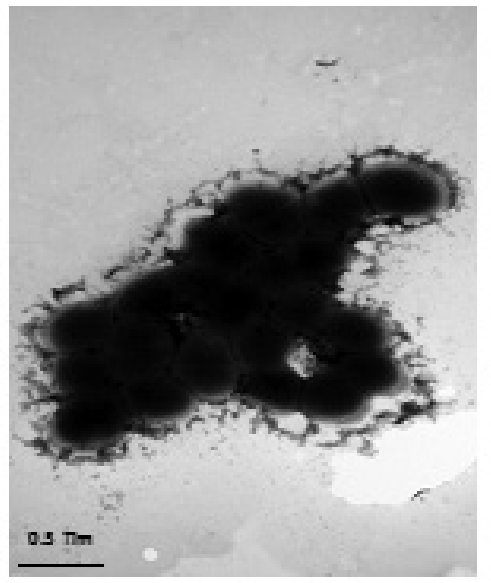

(B)

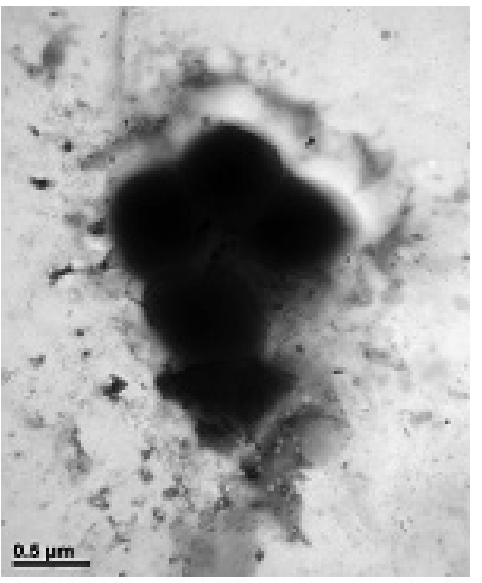

(C)

Figure 6. Transmission electron microscopy images of sophoraflavanone G-treated E. faecium cells. (A) Untreated control E. faecium cells. (B, C) E. faecium cells treated with sophoraflavanone $\mathrm{G}(6.25 \mu \mathrm{g} / \mathrm{ml} 1 / 2 \mathrm{MIC})$.

sophoraflavanone $\mathrm{G}$ induced cell lysis and release of cytoplasmic contents (Fig. 6b and c) compared with untreated normal bacterial cells (Fig. 6a).

\section{DISCUSSION}

The occurrence of resistant strains to antibiotics has become a problem of the world because of the extended duration of treatment and cost. Enterococci commonly showed the intrinsic or acquired resistance to most of the antibiotics used in humans (Climo et al., 1999).

Sophoraflavanone $\mathrm{G}$ is a component extensively found in medicinal plants and has been used to treat the atopic dermatitis and more serious medical issues like malaria and myeloid leukemia. In particular, Sophora species containing sophoraflavanone G showed an antibacterial activity against $S$. aureus, $S$. mutans, Porphyromonas gingivalis, and S. sobrinus (Gerber et al., 2009; Hsiang et al., 2001; Kuroyanagi et al., 1999; Orsi et al., 2011; Tsai et al., 2004).

This study showed that sophoraflavanone $\mathrm{G}$ inhibited $E$. faecium growth by disturbing bacterial cell walls. Sophoraflavanone $\mathrm{G}$ showed the highest stable antibacterial activity compared with acetylshikonin, 6,8-diprenylorobol, kurarinone, pimaric acid, and corosolic acid, which were used to treat E. faecium (Table 1).

The membrane perturbing effect of some of the antimicrobial flavanones was studied in association with a reduction of membrane fluidity. Accordingly, sophoraflavanone $\mathrm{G}$ is regarded to decrease the integrity of the cell wall, and this is confirmed by treatment of Triton X-100 that accelerates the membrane perturbing the activity of sophoraflavanone $G$ (Lee et al., 2015; Mun et al., 2014). PGN is the major component of the cell wall of gram-positive bacteria including E. faecium and a substantial part of osmotic conservation. As shown in Figure 5, sophoraflavanone $\mathrm{G}(12.5 \mu \mathrm{g} / \mathrm{ml})$ showed anti-E. faecium activity by binding to $\mathrm{PGN}$, and this suggests that direct binding of sophoraflavanone $\mathrm{G}$ to the bacterial PGN induced damage of the bacterial cell wall. TEM images of sophoraflavanone G-treated $E$. faecium also confirmed the disruption of the cell wall of $E$. faecium by sophoraflavanone G (Lee et al., 2015; Mun et al., 2014).
This study provided evidence that sophoraflavanone $\mathrm{G}$ possesses anti-E. faecium activity by disrupting the cell wall through binding to PGNs. Therefore, sophoraflavanone G should be a good candidate to treat multidrug-resistant $E$. faecium infection. However, more studies including toxicity, bioavailability, and combination tests with existing antibiotics are required to confirm the availability as an antibacterial agent.

\section{CONFLICT OF INTEREST}

Authors declared that there is no conflicts of interest.

\section{FUNDING}

None.

\section{REFERENCES}

Climo MW, Patron RL, Archer GL. Combinations of vancomycin and beta-lactams are synergistic against staphylococci with reduced susceptibilities to vancomycin. Antimicrob Agents Chemother, 1999; 43(7):1747-53.

Clinical and Laboratory Standards Institute. Methods for dilution antimicrobial susceptibility tests for bacteria that grow aerobically. Approved standards, document M7-A5, Clinical and Laboratory Standards Institute (CLSI), Wayne, PA, 2006.

Desmarais MS, Cava F, Pedro MAD, Huang KC. Isolation and preparation of bacterial cell walls for compositional analysis by ultraperformance liquid chromatography. J Vis Exp, 2014; (83):e51183.

Furukawa H, Tsay JT, Jackowski S, Takamura Y, Rock CO. Thiolactomycin resistance in Escherichia coli is associated with the multidrug resistance efflux pump encoded by emrAB. J Bacteriol, 1993; 175(12):3723-9.

Gerber JS, Coffin SE, Smathers SA, Zaoutis TE. Trends in the incidence of methicillin-resistant Staphylococcus aureus infection in children's hospitals in the United States. Clin Infect Dis, 2009; 49(1):65-71.

Graham JC, Gould FK. Role of aminoglycosides in the treatment of bacterial endocarditis. J Antimicrob Chemother, 2002; 49(3):437-44.

Hsiang CY, Hsieh CL, Wu SL, Lai IL, Ho TY. Inhibitory effect of anti-pyretic and anti-inflammatory herbs on herpes simplex virus replication. Am J Chin Med, 2001; 29(3-4):459-67.

Jorgensen JH, Ferraro MJ. Antimicrobial susceptibility testing: a review of general principles and contemporary practices. Clin Infect Dis, 2009; 49(11):1749-55. 
Kim YC, Kim HS, Wataya Y, Sohn DH, Kang TH, Kim MS, Kim YM, Lee GM, Chang JD, Park H. Antimalarial activity of lavandulyl flavanones isolated from the roots of Sophora flavescens. Biol Pharm Bull, 2004; 27(5):748-50.

Kuhner D, Stahl M, Demircioglu DD, Bertsche U. From cells to muropeptide structures in 24h: peptidoglycan mapping by UPLC-MS. Sci Rep. 2014; 4:7494.

Kuroyanagi M, Arakawa T, Hirayama Y, Hayashi T. Antibacterial and antiandrogen flavonoids from Sophora flavescens. J Nat Prod, 1999; 62(12):1595-9.

Lee YS, Lee DY, Kim YB, Lee SW, Cha SW, Park HW, Kim GS, Kwon DY, Lee MH, Han SH. The mechanism underlying the antibacterial activity of shikonin against methicillin-resistant Staphylococcus aureus. Evid Based Complement Alternat Med, 2015; 2015:520578.

Lim D, Strynadka NC. Structural basis for the $\beta$-lactam resistance of PBP2a from methicillin-resistant Staphylococcus aureus. Nat Struct Biol, 2002; 9(11):870-6.

Miller WR, Munita JM, Arias CA. Mechanisms of antibiotic resistance in Enterococci. Expert Rev Anti Infect Ther, 2015; 12(10): $1221-36$.

Morrison D, Woodford N, Cookson B. Enterococci as emerging pathogens of humans. Soc Appl Bacteriol Symp Ser, 1997; 26:89S-99S.

Mun SH, Joung DK, Kim SB, Park SJ, Seo YS, Gong R, Choi JG, Shin DW, Rho JR, Kang OH, Kwon DY. The mechanism of antimicrobial activity of sophoraflavanone B against methicillin-resistant Staphylococcus aureus. Foodborne Pathog Dis, 2014; 11(3):234-9.

Murdoch DR, Mirrett S, Harrell LJ, Monahan JS, Reller LB. Sequential emergence of antibiotic resistance in enterococcal bloodstream isolates over 25 years. Antimicrob Agents Chemother, 2002; 46(11):3676-8.

Murray BE. The life and times of the enterococcus. Clin Microbiol Rev, 1990; 3(1):46-65.
Orsi GB, Falcone M, Venditti M. Surveillance and management of multidrug-resistant microorganisms. Expert Rev Anti-Infect Ther, 2011; 9(8):653-79.

Sasaki H, Kashiwada Y, Shibata H, Takaishi Y. Prenylated flavonoids from Desmodium caudatum and evaluation of their anti-MRSA activity. Phytochemistry, 2012; 82:136-42.

Septama AW, Xiao J, Panichayupakaranant P. A synergistic effect of artocarpanone from Artocarpus heterophyllus L. (Moraceae) on the antibacterial activity of selected antibiotics and cell membrane permeability. J Intercult Ethnopharmacol, 2017; 6(2):186-91.

Tsai JC, Tsai S, Chang WC. Effect of ethanol extracts of three Chinese medicinal plants with anti-diarrheal properties on in transport of the rat intestinal epithelia. J Pharmacol Sci, 2004; 94(1):60-6.

Vignaroli C, Zandri G, Aquilanti L, Pasquaroli S, Biavasco F. Multidrug-resistant Enterococci in animal meat and faces and co-transfer of resistance from an Enterococcus durans to a human Enterococcus faecium. Curr Microbiol, 2010; 62(5):1438-47.

Viktoryia Z, Vitaly O. Method of isolation of peptidoglycans as a basis for measuring murein-destroying activity of blood serum. J Microbio Exp, 2014; 1(4):144-146.

How to cite this article:

Kim D, Kim KY. Antibacterial effect of sophoraflavanone G by destroying the cell wall of Enterococcus faecium. J Appl Pharm Sci, 2020; 10(09):059-064. 